\title{
Tabaquismo en profesionales de la salud del Instituto Mexicano del Seguro Social, Morelos
}

\author{
Jorge Salmerón-C astro, D r en $C,{ }_{1}^{(1)}$ Edna A rillo-Santillán, M en $C{ }_{1}^{(2)}$ Julio C ésar C ampuzano-Rincón, MSP, ${ }^{(3)}$ \\ Francisco J López-Antuñano, MSP, ${ }^{(3)}$ Eduardo C Lazcano-Ponce, D r en C. ${ }^{(3)}$
}

\section{Salmerón-Castro J,Arillo-Santillán E, Campuzano-Rincón JC, López-Antuñano FJ, Lazcano-Ponce EC. \\ Tabaquismo y profesionales de la salud del Instituto Mexicano del Seguro Social, Morelos. Salud Publica Mex 2002;44 supl 1:S67-S75.} El texto completo en inglés de este artículo está disponible en: http://www.insp.mx/salud/index.html

\section{Resumen}

Objetivo. Determinar la prevalencia de hábito tabáquico por categoría laboral en trabajadores de la salud del Instituto Mexicano del Seguro So cial (IMSS), en el estado de Morelos, México. Material y métodos Se realizó un estudio transversal en 3133 empleados de los servicios médicos de las 23 unidades médicas con que cuenta la delegación Morelos del IMSS, durante el perio do de octubre de 1998 a marzo de 2000. Mediante un cuestionario autoaplicable se recolectó información sobre patrones de consumo de tabaco en distintas etapas de la vida, así como algunas características demográficas y categoría laboral. Se estimaron prevalencias e intervalos de confianza al $95 \%$ por grupos de edad y sexo para distintas categorías laborales. Resulta dos De los 3133 empleados participantes, 53.4\% (IC 95\% 50.8-56.8) de los hombres y $27.4 \%$ (IC 95\% 25.4-29.3) de las mujeres contaban con historia de tabaquismo en algún momento de la vida. La prevalencia de tabaquismo activo fue de $28.3 \%$ (IC 95\% 25.6-31.0) y $14.4 \%$ (IC 95\% 12.815.9) en hombres y mujeres, respectivamente. Entre los hombres, los médicos presentan una prevalencia de tabaquismo activo menor (20.9\%) que la observada entre trabajadores de otras áreas: personal administrativo (33.0\%) y personal técnico de distintas categorías (26.6\%). Las mujeres muestran en general una prevalencia menor que los

\author{
Salmerón-Castro J,Arillo-Santillán E, \\ Campuzano-Rincón JC, López-Antuñano FJ, \\ Lazcano-Ponce EC. \\ Tobacco smoking in healthcare workers \\ of Instituto Mexicano del Seguro Social. \\ Salud Publica Mex 2002;44 suppl 1:S67-S75. \\ The English version of this paper \\ is available at: http://www.insp.mx/salud/index.html
}

\begin{abstract}
A bstract
Objective. To assess the prevalence of tobacco smoking by work type among healthcare workers of Instituto Mexicano del Seguro Social (Mexican Institute of Social Security, IMSS), in Morelos State, Mexico. Material and Methods. A cross-sectional was conducted in 3133 healthcare workers in the 23 medical units of IMSS Morelos District, from 0 ctober 1998 to March 2000. D ata were collected using a self-applied questionnaire on tobacco smoking for different life-stages, so me demo graphic characteristics, and work type. The prevalence rates of smoking and $95 \%$ confidence intervals (95\% CI) were estimated by age groups, gender, and work type. Results. A total of 3133 employees participated; $53.4 \%(95 \% \mathrm{Cl} 50.8-56.8)$ of men and $27.4 \%(95 \% \mathrm{Cl}$ 25.4-29.3) of women reported having smoked tobacco so me time in their lives. The prevalence of current smoking was $28.3 \%(95 \% \mathrm{Cl} 25.6-31.0)$ and $14.4 \%(95 \% \mathrm{Cl} 12.8-15.9)$ in men and women, respectively. A mong men, physicians have a lower prevalence $(20.9 \%)$ of current smoking than that of other workers; for example, the prevalence was $26,6 \%$ among technicians and $33 \%$ among administrative personnel. Women in general had a lower prevalence than men in all categories, and in nurses it was still lower $\mathrm{t}(12,5 \%)$ than that of female physicians (16\%). Conclusions. The prevalence of tobacco smoking in healthcare workers of IMSS
\end{abstract}

Este estudio fue financiado por la Coordinación de investigación en salud del IMSS y el Instituto N acional de Salud Pública.

(1) Unidad de Investigación Epidemiológica y en Servicios de Salud, Instituto Mexicano del Seguro Social, Cuernavaca, Morelos, México.

(2) Secretaría A cadémica. Instituto N acional de Salud Pública, Cuernavaca, Morelos, México.

(3) Centro de Investigación en Salud Poblacional. Instituto N acional de Salud Pública, Cuernavaca, Morelos, México.

Fecha de recibido: 3 de agosto de 2001 • Fecha de aprobado: 14 de diciembre de 2001

Solicitud de sobretiros: D r. Eduardo Lazcano-Ponce. Instituto N acional de Salud Pública.Avenida Universidad 655, colonia Sta. MaríaA huacatitlán, 62508 Cuernavaca, Morelos, México.

Correo electrónico: elazcano@ correo.insp.mx 
hombres en todas las categorías, y las enfermeras presentan una prevalencia discretamente menor $(12.5 \%)$ que las médicas (16.0\%). Conclusiones. El patrón del consumo de tabaco observado en profesionales de la salud del IMSS Morelos muestra una prevalencia menor a la observada en población general. Sin embargo, es necesario continuar con programas para desalentar el consumo del tabaco entre profesionales de la salud, y tratar de hacerlos concientes acerca de la influencia positiva que pueden y deben ejercer en la sociedad para impulsar programas integrados contra el consumo del tabaco y la adicción a la nicotina. El texto completo en inglés de este artículo está disponible en: http://www.insp.mx/salud/index.html

Palabras clave: tabaquismo; profesionales de la salud; México
Morelos was lower than that of the general population. $\mathrm{N}$ evertheless, it is necessary to keep working on tobacco control programs to encourage smoking cessation among healthcare workers and make them aware of the positive role they may play in promoting programs to stop tobacco smoking and nicotine addiction. The English version of this paper is available at: http://ww w.insp.mx/salud/index.html

Key words: smoking; health professionals; Mexico
E l estudio del tabaquismo en profesionales de la salud constituyó el inicio de un nuevo paradigma en la investigación epidemiológica cuando, Doll y Hill, al inicio de la década de los años 50, establecieron una relación causa-efecto entre el consumo de tabaco y la ocurrencia de cáncer pulmonar, mediante un estudio de cohorte en médicos ingleses. ${ }^{1}$ A cincuenta años de este hecho, se estima que existen más de mil millones de fumadores en el mundo, lo cual ocasiona seis muertes por minuto, y se esperan más de diez millones de muertes atribuibles al consumo de tabaco en los próximos 20 años. ${ }^{2}$

Actualmente, la Organización Mundial de la Salud (OMS) promueve en forma prioritaria que el personal médico y paramédico deje de fumar, porque ellos desempeñan una importante función como educadores en la promoción de comportamientos saludables en la población general. Se ha documentado ampliamente la influencia de los médicos y enfermeras en programas de cesación de consumo de tabaco en sus pacientes. ${ }^{3,4}$ Por otra parte, existen diversos reportes que muestran que en promedio el profesional de la salud que tiene el hábito de fumar, no ofrecerá el consejo médico a los pacientes sobre los riesgos del tabaquismo, ${ }^{5-7}$ ni se perciben como ejemplo. ${ }^{8}$

La prevalencia de consumo de tabaco entre los profesionales de la salud en países desarrollados es menor a la observada en la población general. Estados Unidos de América (EUA) cuenta con una prevalencia de tabaquismo entre médicos seis veces menor que la observada en la población general., ${ }^{9} 10$ Por el contrario, en algunos países en desarrollo, en particular para aquellos que no han logrado regular el consumo de tabaco en edificios y lugares públicos, la prevalencia de ta- baquismo en profesionales de la salud resulta igual o mayor que la de la población general. ${ }^{11,12}$

En México son escasos los estudios que determinan la prevalencia de consumo de tabaco por categoría laboral, utilizando como unidad de observación los profesionales de la salud. Este estudio describe la prevalencia de fumadores de tabaco en los trabajadores del IMSS delegación Morelos.

\section{Material y métodos}

Se trata de un estudio transversal cuya población base estuvo constituida por los 5706 trabajadores de la salud del IMSS-Morelos, a quienes se invitó a participar, durante el periodo de octubre de 1998 a marzo de 2000. Un total de 3822 empleados de las áreas médicas y administrativas de las 24 unidades médicas existentes en la institución aceptaron participar en el estudio (67\% de la población base). El análisis se efectuó en 3133 trabajadores (82\% de la población de estudio) con información completa sobre las variables de interés.

Mediante cuestionarios autoaplicables se recogió información sobre características sociodemográficas -edad, sexo y categoría laboral- y consumo de tabaco. Los cuestionarios se codificaron manualmente y se procesaron por lectura óptica.

Se definió el tabaquismo activo, como el consumo de aquellos individuos que al ser entrevistados refirieron fumar actualmente y además tener el antecedente de haber fumado 100 cigarrillos o más en toda su vida. Esta es la categorización propuesta por la $\mathrm{OMS}^{13}$ para definir a la población de fumadores. Cabe mencionar que dicha definición es la misma que se utilizó en la Encuesta Nacional de Salud. ${ }^{14}$ 
La categoría de historia de tabaquismo se construyó agrupando a los ex fumadores y a los fumadores actuales. Asimismo, se construyó un índice de abandono del hábito tabáquico, a partir de la proporción de ex fumadores sobre el total de aquellos sujetos que alguna vez en su vida habían sido fumadores.

Las categorías laborales fueron organizadas en cuatro grupos: 1) auxiliares de administración, auxiliares universales de oficina, auxiliares de farmacia y auxiliares de intendencia; 2) personal de enfermería y el grupo de asistentes médicas; 3 ) médicos familiares y médicos no familiares; y 4) técnicos polivalentes, el personal de nutrición y laboratorio, y retirados.

Se estimaron prevalencias puntuales con sus correspondientes intervalos de confianza al 95\%, para historia de tabaquismo y tabaquismo activo, así como medias de edad de inicio de consumo de tabaco por grupos de edad, sexo y categorías laborales. Se evaluaron diferencias de proporciones entre las prevalencias de tabaquismo para distintas categorías laborales. Para el análisis de los datos se utilizó el paquete estadístico STATA.

\section{Resultados}

Se entrevistó un total de 3133 empleados, 399 médicos, 887 enfermeras y asistentes médicas 1847 trabajadores de diversas categorías laborales. Los sujetos de la población base que no participaron en el estudio $(33 \%)$, no mostraron diferencias con la muestra bajo estudio de acuerdo con sexo, edad, estado civil y escolaridad (datos no mostrados).

La prevalencia global de historia de tabaquismo fue de $36.6 \%$, con una prevalencia de $53.4 \%$ (IC 95\% $50.8-56.8$ ) y $27.4 \%$ (IC 95\% 25.4-29.3) en hombres y mujeres, respectivamente. El tabaquismo activo en toda la población fue de $19.2 \%$, con una frecuencia dos veces superior en hombres que en mujeres ( 28.3 vs $14.4 \%$ ) (cuadro I).

Para uno u otro sexo, se observó una mayor prevalencia de historia de tabaquismo entre los grupos de mayor edad. Tanto en hombres como en mujeres el grupo de edad con mayor prevalencia de tabaquismo activo fue el de 36 a 42 años (30.6 y 16.7\%, respectivamente), como se muestra en la figura 1 .

La edad de inicio de consumo de tabaco es más tardía en mujeres que en hombres, mostrando ambos grupos un claro patrón de inicio más temprano en el hábito tabáquico entre las cohortes de nacimiento más jóvenes.

Con relación a las distintas categorías laborales, entre las mujeres el personal de enfermería muestra una menor prevalencia de tabaquismo activo (12.5\%;

\section{Cuadro I \\ Características sociodemográficas asociadas CON CONSUMO DE TABACO EN 3133 TRABAJADORES del Instituto Mexicano del Seguro Social, Morelos. México, 1998-2000}

\begin{tabular}{|c|c|c|c|c|c|}
\hline \multicolumn{6}{|c|}{ M ujeres (2045) } \\
\hline & & Historia d & e tabaquismo* & * Tabaqu & uismo activo \\
\hline Variables & $\mathrm{N}$ & Prevalenci & & & ía $\quad$ IC al $95 \%$ \\
\hline \multicolumn{6}{|l|}{ Edad } \\
\hline$<36$ años & 587 & 22.1 & $(18.8,25.7)$ & 13.5 & $(10.8,16.4)$ \\
\hline 36 a 42 años & 617 & 29.3 & $(25.7,33.1)$ & 16.7 & $(13.8,19.8)$ \\
\hline 43 a 48 & 511 & 28.8 & $(24.8,32.9)$ & 12.3 & $(9.6,15.4)$ \\
\hline 48 a 80 & 330 & 30.9 & $(25.9,36.2)$ & 14.9 & $(11.1,19.1)$ \\
\hline Total & 2045 & 27.4 & $(25.4,29.3)$ & 14.4 & $(12.8,15.9)$ \\
\hline \multicolumn{6}{|l|}{ Adscripción } \\
\hline Zacatepec & 321 & 22.4 & $(17.9,27.3)$ & 13.4 & $(9.8,17.6)$ \\
\hline Cuautla & 442 & 28.7 & $(24.5,33.1)$ & 14.9 & $(11.7,18.6)$ \\
\hline Clínica 20 & 431 & 23.2 & $(19.2,27.4)$ & 9.3 & $(6.7,12.4)$ \\
\hline Hospital HGR1 & 1851 & 30.7 & $(27.5,33.8)$ & 17.0 & $(14.5,19.7)$ \\
\hline Total & 2045 & 27.4 & $(25.4,29.3)$ & 14.4 & $(12.8,15.9)$ \\
\hline \multicolumn{6}{|l|}{ Categoría trabajo } \\
\hline Categoría 1 & 776 & 26.4 & $(23.3,29.6)$ & 14.9 & $(12.5,17.6)$ \\
\hline C ategoría 2 & 861 & 26.4 & $(23.4,29.4)$ & 12.5 & $(10.4,14.9)$ \\
\hline Categoría 3 & 150 & 34.7 & $(27.0,42.8)$ & 16.0 & $(10.5,22.8)$ \\
\hline Categoría 4 & 258 & 29.5 & $(23.9,35.4)$ & 17.8 & $(13.3,23.0)$ \\
\hline Total & 2045 & 27.4 & $(25.4,29.3)$ & 14.4 & $(12.8,15.9)$ \\
\hline \multicolumn{6}{|c|}{ Hombres (1088) } \\
\hline \multicolumn{6}{|l|}{ Edad } \\
\hline$<36$ años & 243 & 42.4 & $(36.0,48.8)$ & 27.2 & $(21.6,33.2)$ \\
\hline 36 a 42 años & 307 & 53.1 & $(47.3,58.7)$ & 30.6 & $(25.5,36.1)$ \\
\hline 43 a 48 & 311 & 56.9 & $(51.2,62.4)$ & 26.4 & $(21.5,31.6)$ \\
\hline 48 a 80 & 227 & 63.0 & $(56.3,69.2)$ & 29.1 & $(23.2,35.4)$ \\
\hline Total & 1088 & 53.9 & $(50.8,56.8)$ & 28.3 & $(25.6,31.0)$ \\
\hline \multicolumn{6}{|l|}{ Adscripción } \\
\hline Zacatepec & 180 & 50.6 & $(43.0,58.0)$ & 30.6 & $(23.9,37.8)$ \\
\hline Cuautla & 188 & 54.3 & $(46.8,61.5)$ & 28.7 & $(22.3,35.7)$ \\
\hline Clínica 20 & 397 & 53.2 & $(48.1,58.1)$ & 25.9 & $(21.6,30.5)$ \\
\hline Hospital HGR1 & 323 & 56.4 & $(50.7,61.8)$ & 29.7 & $(24.7,35.0)$ \\
\hline Total & 1088 & 53.9 & $(50.8,56.8)$ & 28.3 & $(25.6,31.0)$ \\
\hline \multicolumn{6}{|l|}{ Categoría trabajo } \\
\hline Categoría 1 & 546 & 56.4 & $(52.1,60.6)$ & 33.0 & $(29.0,37.0)$ \\
\hline Categoría 2 & 26 & 46.2 & $(26.5,66.6)$ & 19.2 & $(6.5,39.3)$ \\
\hline Categoría 3 & 249 & 48.2 & $(41.8,54.8)$ & 20.9 & $(16.0,26.4)$ \\
\hline Categoría 4 & 267 & 54.7 & $(48.4,60.7)$ & 26.6 & $(21.3,32.3)$ \\
\hline Total & 1088 & 53.9 & $(50.8,56.8)$ & 28.3 & $(25.6,31.0)$ \\
\hline
\end{tabular}

Categoría 1: auxiliares de administración, auxiliares universales de oficina, auxiliar de farmacia, personal administrativo, auxiliares de intendencia, auxiliar trabajo social y educación física

Categoría 2: enfermería, asistentes médicas

Categoría 3: médicos familiar y no familiar

Categoría 4: técnicos polivalentes, estudiantes, nutrición, laboratorio y retirados

HGRI: Hospital General Regional N 0.1 

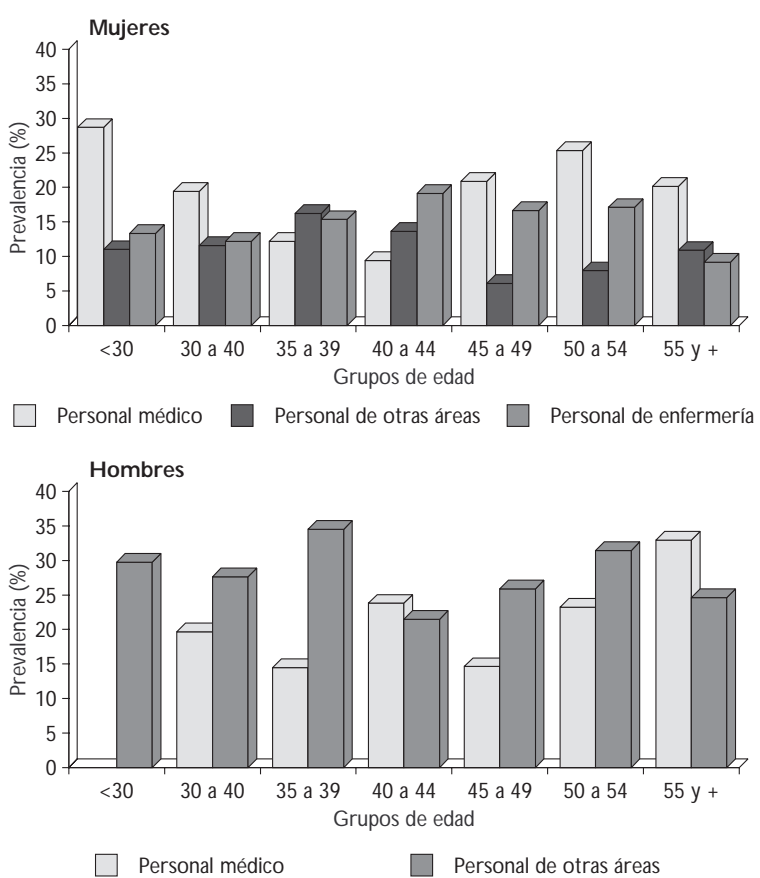

Figura 1. Prevalencia de tabaquismo activo por CATEgoría de tRABAJO, EDAD Y SEXO. INSTITUTO Mexicano del Seguro Social, Morelos. México, 1998-2000

IC 95\% 10.4-14.9), en comparación con médicas (16.0\%; IC 95\% 10.5-22.8), personal técnico (17.8\%) o administrativo (13.5\%), sin embargo, estas diferencias no fueron estadísticamente significativas. Entre los hombres, el tabaquismo activo en personal de enfermería fue significativamente menor al resto de trabajadores del mismo género (19.2 \% vs 20.9\% (médicos), $26.6 \%$ (técnicos), a $33.0 \%$ (administrativo); $p<0.05$ ).

Respecto al índice de abandono, el personal médico presentó una proporción mayor de abandono que el resto de las categorías laborales ( $55.8 \%$ vs 47.8 ) siendo más evidente en el caso de los médicos (56.6\%), como se obseva en el cuadro II.

\section{Discusión}

En médicos del IMSS de la delegación Morelos, la prevalencia de consumo de tabaco es menor que en otras categorías laborales. El consumo observado en médicos morelenses es similar al descrito en encuestas recientes en otras instituciones de salud y aproximadamente menor $30 \%$ a la prevalencia reportada en la población general (cuadro III).

\section{Cuadro II \\ INDICE DE ABANDONO DEL TABAQUISMO EN PERSONAL de salud del Instituto Mexicano del Seguro Social, Morelos. México, 1998-2000}

\begin{tabular}{lccc} 
& Mujeres (\%) & Hombres (\%) & Total (\%) \\
Categoría 1 & 44.9 & 42.8 & 43.7 \\
\hline Categoría 2 & 52.4 & & 52.4 \\
\hline Categoría 3 & 53.8 & 56.6 & 55.8 \\
\hline Categoría 4 & 39.5 & 51.4 & 47.3 \\
\hline Total & 48.0 & 48.1 & 48.1
\end{tabular}

El índice de abandono se calculó dividiendo a la población de ex fumadores sobre el total de población que ha tenido contacto con el tabaco (ex fumadores + fumadores actuales) $* 100$

Categoría 1: auxiliares de administración, auxiliares universales de oficina, auxiliar de farmacia, personal administrativo, auxiliares de intendencia, auxiliar trabajo social y educación física Categoría 2: enfermería, asistentes médicas.

Categoría 3: médicos familiar y no familiar

Categoría 4: técnicos polivalentes, estudiantes, nutrición, laboratorio y retirados

La prevalencia de tabaquismo activo, como de historia de tabaquismo, es superior en hombres con relación a las mujeres. Similares resultados han mostrado encuestas realizadas en profesionales médicos en otros países, como es el caso de Japón ${ }^{6}$ (mujeres $6.8 \%$, hombres $27.1 \%$ ), China $^{15}$ (mujeres $12.2 \%$, hombres $61.3 \%$ ), Francia ${ }^{16}$ (mujeres $24 \%$, hombres $33 \%$ ), Italia ${ }^{17}$ (mujeres $29 \%$, hombres $34 \%$ ), Holanda ${ }^{18}$ (mujeres $24 \%$, hombres $41 \%$ ). Cabe mencionar que se ha dado una liberalización de las normas concernientes a los comportamientos de la mujer, que contribuye a una progresiva aceptación social del consumo de tabaco por parte de ellas. ${ }^{19}$ De acuerdo con algunos estudios de tendencias internacionales, se espera un aumento en el consumo de tabaco, en el grupo de mujeres,$^{20}$ como ya se evidencia en ciertos países. ${ }^{9,16}$

Respecto al consumo por grupo de edad, en ambos sexos, se observa una mayor prevalencia de historia de tabaquismo en los grupos de mayor edad.

Existen estudios que han mostrado una baja prevalencia de consumo en estudiantes de medicina respecto a sus pares de otras áreas académicas, lo que podría sugerir que en la nueva generación el personal médico y de enfermería, puede desempeñar un papel más activo como ejemplo. En este contexto, existen autores que proponen como parte de una política antitabáquica, que la prevención del hábito tabáquico debería formar parte del currículum de las escuelas de medicina. ${ }^{18}$ 


\section{Prevalencia de tabaquismo en Personal médico de distintos países}

\begin{tabular}{|c|c|c|c|c|c|c|c|c|}
\hline \multirow[b]{2}{*}{ País } & \multirow[b]{2}{*}{ Año } & \multirow[b]{2}{*}{ Profesión } & \multicolumn{3}{|c|}{$\begin{array}{l}\text { Tabaquismo activo. } \\
\text { Médicos }\end{array}$} & \multicolumn{3}{|c|}{$\begin{array}{l}\text { Tabaquismo activo. } \\
\text { Población general }\end{array}$} \\
\hline & & & Total & Hombres & M ujeres & Año & Hombres & M ujeres \\
\hline Filipinas & 1987 & Médicos & 63.0 & - & - & 1999 & 75 & 18 \\
\hline China & 1996 & Médicos & - & 61.3 & 12.2 & 1996 & 63 & 3.8 \\
\hline Bosnia & 1996 & Médicos & - & 55 & 50 & 1995 & $48(\mathrm{amb}$ & xos) \\
\hline Turquía & 1993 & Médicos & - & 52.9 & 41.0 & 1997 & 51 & 49 \\
\hline Bulgaria & 1996 & Médicos & 52.3 & - & - & 1996 & 49.2 & 23.8 \\
\hline Grecia & 1992 & Médicos & - & 50.0 & 16.8 & 1994 & 46 & 28 \\
\hline Portugal & 1991 & Médicos & - & 44 & - & $1995-96$ & 30.2 & 7.1 \\
\hline República D ominicana & 1986 & Médicos & - & 43 & 16.9 & 1993 & 24.3 & 17.1 \\
\hline Holanda & 1989 & Médicos & - & 41 & 24 & 1998 & 37 & 30 \\
\hline Hungría & 1995 & Cirujanos & 41 & - & - & $1998-99$ & 44 & 27 \\
\hline Rusia & 1996-99 & Médicos & - & 41 & 13.0 & 1996 & 63 & 14 \\
\hline Chile & 1992 & Médicos & - & 40.2 & 23.8 & 1998 & 26 & 18.3 \\
\hline Rumania & 1997 & Médicos & - & 40 & 19 & 1994 & 42.7 & 15.2 \\
\hline España & 1995 & Médicos & - & 37.4 & 31.6 & 1997 & 42.1 & 24.7 \\
\hline$\overline{\text { Argelia }}$ & 1999 & Estudiantes & 37 & - & - & $1997-98$ & 43.8 & 6.6 \\
\hline Argentina & 1997 & Médicos & 30.3 & - & 36.3 enfermeras & 1999 & 46.8 & 34.0 \\
\hline Guatemala & 1987 & Médicos y estudiantes & - & 34 & 36 & 1989 & 37.8 & 17.7 \\
\hline Paraguay & 1889 & Médicos & - & 35.2 & 23.9 & 1990 & 24.1 & 5.5 \\
\hline Bolivia & 1987 & Médicos & 35.0 & - & - & 1998 & 42.7 & 18.1 \\
\hline Italia & 1996 & Médicos & - & 29 & 34 & 1998 & 32.2 & 17.3 \\
\hline Uruguay & 1992 & Profesionales de la salud & 30 & - & - & 1995 & 31.7 & 14.3 \\
\hline Alemania & $1992-93$ & Estudiantes de medicina & - & 29.2 & 17.6 & 1997 & 43.2 & 30 \\
\hline Cuba & 1995 & Médicos & 25 & - & 29 enfermeras & 1995 & 48 & 26.3 \\
\hline Japón & 1990 & Médicos & 24.8 & - & - & 1998 & 52.8 & 13.4 \\
\hline Dinamarca & 1996 & Médicos & - & 29 & 20 & 1998 & 32 & 30 \\
\hline Bélgica & 1991 & Médicos & - & 28.7 & 16.5 & 1999 & 31 & 26 \\
\hline República Checa & 1998 & Médicos & - & 26 & 21 & 1998 & 28 & 12 \\
\hline Polonia & 1995 & Médicos & - & 24 & 16 & 1998 & 39 & 19 \\
\hline Canadá & 1994 & $\begin{array}{l}\text { Médicos y profesionales } \\
\text { de la salud }\end{array}$ & 22 & - & - & 1999 & 27 & 23 \\
\hline Colombia & 1991 & Médicos & - & 21.2 & 21.7 & 1997 & 23.5 & 21.0 \\
\hline Suiza & 1989 & Médicos & - & 20.1 & 16.2 & 1997 & 38.4 & 27.4 \\
\hline Sur-A frica & 1986 & Anestesiólogos & 19 & - & - & 1998 & 42 & 11 \\
\hline Costa Rica & 1993-94 & Médicos & 19.0 & - & - & 1995 & 28.6 & 6.6 \\
\hline Panamá & 1980 & Médicos & 18.7 & - & - & 1993 & 56 & 20 \\
\hline EUA & 1991 & Médicos & 3.3 & - & 18.3 enfermeras & 1997 & 27.6 & 22.1 \\
\hline Tailandia & 1989 & Profesionales de la salud & 17.1 & - & - & 1999 & 38.9 & 2.4 \\
\hline Francia & 1993 & Cardiólogos & - & 13 & 17 & 1997 & 39 & 27 \\
\hline Inglaterra & 1985-97 & Médicos & - & 13 & 5.4 & 1996 & 29 & 28 \\
\hline Israel & $1995-96$ & Médicos & 16 & - & - & 1999 & 33 & 25 \\
\hline Venezuela & 1990 & Residentes de medicina & 15.7 & - & - & 1992 & 41.8 & 39.2 \\
\hline Noruega & 1993 & Médicos & - & 14 & 8 & 1998 & 33.7 & 32.3 \\
\hline Finlandia & 1995 & Médicos & - & 7.0 & 3.0 & 1999 & 27 & 20 \\
\hline Suecia & 1996 & Médicos & - & 6 & 6 & 1998 & 17.1 & 22.3 \\
\hline México & 1997 & Médicos & 22 & - & - & 1998 & 51.2 & 18.4 \\
\hline Brasil & 1996 & Médicos & 6.4 & - & - & 1995 & 38.2 & 29.3 \\
\hline Australia & 1996 & Médicos & - & 4.2 & 1.6 & 1995 & 27.1 & 23.2 \\
\hline India & 1985 & Estudiantes & - & 2.7 & 0.0 & $1985-86$ & 45 & 7 \\
\hline
\end{tabular}

Fuente:Tobacco free initiative. Statistics W orld Health O rganization. Ginebra, julio 1999 
Los profesionales de la salud pueden ejercer una enorme influencia en cuanto al tabaquismo de la población general, debido a que pueden desalentar el consumo de diversas maneras. Pueden servir como ejemplo o modelo, promoviendo estilos de vida saludables, fomentando el ejercicio de la legislación sobre la prohibición del consumo de tabaco en las unidades médicas, y sensibilizando a la sociedad sobre los problemas derivados del tabaquismo. ${ }^{21,22}$

Diversos estudios documentan que los médicos que no fuman muestran un mayor interés en desalentar a sus pacientes fumadores, en comparación con los médicos que fuman. ${ }^{5,6}$ Tradicionalmente se ha concebido que el personal médico tiene conocimiento de las diversas causas de enfermedad y ha aprendido a reconocer el riesgo de eventos no saludables en sus pacientes, sin embargo, no siempre reconoce su propio riesgo de enfermar o morir. ${ }^{23}$ Nagle y colaboradores informaron que un porcentaje importante de los médicos no contaban con conocimientos óptimos sobre los riesgos que conlleva el hábito tabáquico y desconocían posibles tratamientos; en personal de enfermería la situación es similar. ${ }^{23}$

Los médicos que reconocen fumar activamente, no se perciben como un ejemplo para la población general respecto a la prevención de consumo de tabaco, 5,8 y algunos estudiantes de medicina tienen un bajo reconocimiento de su función social como ejemplo en el cambio de conducta. Este fenómeno existe a pesar de que se ha señalado en reportes previos que un porcentaje importante de sujetos han dejado de fumar debido a la influencia de algún profesional de la salud. ${ }^{4}$ El consejo educativo, intervenciones conductuales y terapias farmacológicas, tanto de personal médico como de enfermería, han demostrado ser costo efectivas para cesación del consumo de tabaco. ${ }^{26}$

Con la información obtenida de diferentes encuestas de prevalencia de tabaquismo en médicos en varios países (cuadro III), se podrían establecer de manera general tres patrones de consumo. Uno de ellos, revela una prevalencia de tabaquismo mayor entre los profesionales de la salud, en comparación con la población en general. En países como Filipinas, China, Bosnia, Turquía, Bulgaria y Grecia, entre otros, se presentan las más altas prevalencias de consumo de tabaco en profesionales de la salud. Diversos factores pueden estar influyendo en este fenómeno. Por ejemplo, la creencia de que el cigarrillo alivia el estrés ocasionado por el trabajo en clínicas o situaciones adversas, como el caso de médicos de Bosnia y Herzegovina, que durante los últimos años sufrieron la angustia de la guerra. Asimismo, el incremento de la oferta de cigarrillos por compañías trasnacionales en los países de referencia, ${ }^{27}$ y la escasa restricción del tabaquismo en espacios públicos, ${ }^{11}$ contribuyen en el exceso de consumo.

El segundo patrón corresponde a prevalencias de consumo de tabaco en médicos, similares a las reportadas en la población general. Esta situación se presenta en países como Rumania, donde se ha reportado que más de $40 \%$ de los médicos son fumadores y que $53 \%$ de estos son mujeres. Esto probablemente se relaciona con el hecho de que no existen restricciones en el consumo del tabaco en áreas y edificios públicos, aunado a que el tratamiento para suprimir el hábito, tiene un costo 15 veces mayor que una cajetilla de cigarrillos. ${ }^{12}$ En una muestra de médicos chilenos, se notificó un promedio de nueve cigarrillos por día en $40.2 \%$ de ellos, aunque la mayoría informó no hacerlo en presencia de sus pacientes. ${ }^{28}$ El hábito de fumar tabaco en médicos de los Emiratos Arabes y Kuwait, es cercano a $45 \% .^{29}$ En Brasil, una encuesta nacional realizada en médicos reveló una prevalencia de consumo de tabaco de $34.3 \%$, y la ausencia del hábito se observa principalmente en aquellos cuyas especialidades cuentan con programas de control antitabaco, como son los servicios de neumología, oncología, cardiología y otorrinolaringología. ${ }^{30}$ En médicos españoles la prevalencia de consumo de tabaco es igualmente elevada $(42 \%)$, y cerca de $90 \%$ de los fumadores, al no existir restricciones en edificios públicos, reportan fumar dentro del hospital. ${ }^{11}$

El tercer patrón de consumo, corresponde a aquellos países cuya prevalencia ha disminuido notablemente durante los últimos años; evidenciándose un descenso mayor en personal médico que en personal de enfermería, como lo revelan encuestas en EUA. ${ }^{9,10}$ $(3 \%-10 \%)$, Inglaterra ${ }^{31}(5 \%, 20 \%)$ y Nueva Zelanda ${ }^{32}$ $(5 \%, 18 \%)$. Específicamente en personal de enfermería, Nagle ${ }^{24}$ menciona que es evidente un decremento en el consumo desde la década de los años 70, fenómeno común en varios países. En EU desde hace 25 años el consumo de tabaco ha disminuido rápidamente de una prevalencia de $18.8 \%$ a $3.3 \%$ entre médicos y de $31.7 \%$ a $18.3 \%$ en enfermeras, aunque llama la atención que el consumo por sexo se ha invertido, pues como se observa la prevalencia es mayor en mujeres. ${ }^{9}$

En México, durante los últimos diez años, se han realizado diversas encuestas ${ }^{33-39}$ con el fin de caracterizar la prevalencia de fumadores de tabaco entre trabajadores de la salud (cuadro IV). Estos trabajos han revelado una prevalencia menor de consumo de tabaco en profesionales de la salud con relación a la población general. Los profesionales de la salud estudiados en el IMSS-Morelos, presentan una prevalencia dos 


\section{Cuadro IV \\ Estudios de prevalencia de tabaquismo en personal médico en México}

\begin{tabular}{|c|c|c|c|c|c|c|c|c|}
\hline \multirow[b]{2}{*}{ Autor } & \multirow[b]{2}{*}{ Año } & \multirow[b]{2}{*}{ Institución } & \multirow[b]{2}{*}{ Ocupación } & \multicolumn{5}{|c|}{ Tabaquismo activo } \\
\hline & & & & $\mathrm{N}$ & Total & Mujeres & Hombres & Ex fumadores \\
\hline Ramírez-C asanova y colaboradores ${ }^{36}$ & 1991 & Hospital General de la Ciudad de México & Médicos & 284 & $61.6 \%$ & $20.8 \%$ & $29.9 \%$ & $20.6 \%$ \\
\hline Tapia-C onyer y colaboradores 38 & 1993 & IMSS, ISSSTE y SSA, Ciudad de México & Médicos & 3488 & $26.9 \%$ & & & \\
\hline \multirow[t]{2}{*}{ Labrandero M y colaboradores ${ }^{35}$} & 1994 & IMSS C iudad de México & Médicos & 6061 & $25.3 \%$ & $17.6 \%$ & & $39.5 \%$ \\
\hline & & & y enfermeras & & & nfermera & & \\
\hline \multirow[t]{2}{*}{ Rojo Padilla y colaboradores ${ }^{39}$} & 1998 & ISSSTE & Médicos & 500 & $20.7 \%$ & & & $27.0 \%$ \\
\hline & & & Enfermeras & 214 & $62.6 \%$ & & & \\
\hline \multirow[t]{2}{*}{ Peña Corona y colaboradores ${ }^{37}$} & 1998 & IMSS-36 delegaciones a & Médicos & 5133 & $27.0 \%$ & & & \\
\hline & & nivel nacional & & & & & & \\
\hline \multirow[t]{2}{*}{ Gómez-García R, Grimaldi A ${ }^{38}$} & 1998 & Hospital Materno Infantil & Médicos & 48 & $33.3 \%$ & & & \\
\hline & & Inguarán Ciudad de México & Enfermeras & 79 & $20.0 \%$ & & & \\
\hline Sansores $R$ y colaboradores ${ }^{34}$ & 1998 & Institutos N acionales de Salud, SSA & Médicos & 238 & $22.0 \%$ & & & \\
\hline \multirow[t]{2}{*}{ Salmerón J y colaboradores } & 2000 & IMSS D elegación Morelos & Médicos & 399 & $19.0 \%$ & $16.0 \%$ & $20.9 \%$ & $24.0 \%$ \\
\hline & & & Enfermeras & 887 & & $12.5 \%$ & $12.5 \%$ & \\
\hline
\end{tabular}

IMSS: Instituto Mexicano del Seguro Social

ISSSTE: Instituto de Seguridad y Servicios Sociales de los Trabajadores del Estado

SSA: Secretaría de Salud

veces menor a la reportada en la Encuesta Nacional de Salud en México. ${ }^{14} \mathrm{Y}$ una prevalencia ligeramente menor, respecto a población derechohabiente del IMSS (17.6\% en mujeres y $40 \%$ en hombres) mayor en los estados del norte que en el resto de México. ${ }^{40}$

Los resultados de Morelos son consistentes con diversos estudios que han comprobado que los médicos tienen actualmente una menor predisposición al hábito de fumar tabaco, en comparación con la población general. ${ }^{41}$ Una encuesta aplicada en 6061 médicos y enfermeras del IMSS en el Distrifo Federal, en 1994,33 halló una prevalencia de fumadores activos de $25.3 \%$ en personal médico y $17.6 \%$ en personal de enfermería. Asimismo, en médicos mexicanos de los Institutos Nacionales de Salud, se reportó una prevalencia de fumadores de $22.0 \%$, frecuencia significativamente menor a la de un grupo de fumadores no médicos $(28.0 \%) .{ }^{34}$ Esta prevalencia es similar a la encontrada en 1993, en un estudio de médicos del Sistema Nacional de Salud, con una prevalencia de fumadores activos de $26.9 \%{ }^{35}$ Este hallazgo coincide con el de la Encuesta de Trabajadores del IMSS-Morelos, donde la prevalencia de consumo de tabaco en médicos es de $27.2 \%$, similar a la de la ENA $1998,{ }^{14}$ con una prevalencia de $27.7 \%$ en población total.

Como se observa, la frecuencia de consumo es mayor en profesionales médicos y de enfermería en comparación con la población general, lo que debería capitalizarse en la aplicación de las restricciones vigentes y en la instrumentación de políticas y programas tendientes a reducir el consumo, no únicamente del personal, sino de pacientes y visitantes de los centros de salud.

Cabe mencionar que la información derivada de este estudio proviene de encuestas autoaplicadas que pudieran no corresponder con la frecuencia real de uso de tabaco en la población en estudio. Sin embargo, trabajos previos han documentado una alta correlación entre el reporte por cuestionarios autoaplicables y biomarcadores de consumo de tabaco, con rangos estrechos de clasificación errónea. ${ }^{42}$

Una limitación más de este trabajo obedece a que la población de estudio no constituye una muestra representativa de profesionales de la salud en México y los resultados no pudieran ser generalizados al resto del país, sino únicamente a empleados de los servicios médicos del IMSS, Morelos.

Los profesionales de la salud son agentes fundamentales para promover la agenda de investigación y acción encaminada a combatir la epidemia de tabaco. $^{43}$ Es necesario establecer programas para desalentar el consumo del tabaco en profesionales de la salud, adicionalmente a programas de educación médica continua, actualización, educación en salud y capacitación, ${ }^{44}$ instituidos para motivarlos acerca de la influencia que pueden y deben ejercer en la sociedad. 
Los premisas básicas son concientizar a los médicos y enfermeras acerca de los daños a la salud producidos por la exposición activa y pasiva al humo del cigarrillo; reconocer que la nicotina es una droga adictiva, que requiere regulación; capacitar a los profesionales de la salud en el diagnóstico y tratamiento del paciente fumador. Finalmente, se necesita enfatizar y sensibilizar al personal de salud sobre la responsabilidad social que tiene en la prevención, tratamiento y aplicación de políticas públicas de cesación de consumo de tabaco, ya que deben asumir esta tarea imperativa y éticamente impostergable.

\section{Agradecimientos}

Se agradece la participación de los trabajadores del Instituto Mexicano del Seguro Social en la Delegación Morelos.

\section{Referencias}

1. D oll R, B. Hill A. Smoking a carcinoma of the lung preliminary report. Br Med J 1950;2:739-748.

2. Lazcano-Ponce E, Hernández-Avila M. Cáncer: frecuencia, factores de riesgo y medidas de prevención (editorial). Salud Publica Mex 1997; 39(4):251-225

3. Goldberg RJ, 0 ckene IS, 0 ckenee JK, Mariam P, Kristeller J. Physicians'attitudes and reportes practices toward smoking intervention. J Cancer Educ 1993;8: 133-139.

4. Lancaster T, Silagy C, Fowler G. Training health professionals in smoking cessation. Cochrane Database Syst Rev 2000;(3):C D 000214.

5. Bener A. Gomes J,Anderson JA,A bdullah S. Smoking among health professionals. Med Educ 1994;28:151-157.

6. 0 hidaT, Sakurai H, MochizukiY, Kamal AM, Takemura S, Minowa M et al. Smoking prevalence and attitudes toward smoking among Japanese physicians. JA MA 2001;28:2643-2648.

7. 0 live K, Ballard J.Attitudes of Patients Toward Smoking by Health Professionals. Public Health Rep 1992;107:335-339.

8. Grossman DW, Know JJ, Nash C, Jiménez JG. Smoking: Attitudes of Costa Rican physicians and opportunities for intervention. Bull World Health 0 rgan 1999;77:315-322.

9. N elson DE, Giovino G, Emont S, Brackbill R, Cameron, Peddicord J, Mowery P.Trends in cigarette smoking among US physicians and nurses. JAMA 1994;271(16):1273-1275.

10. Hensrud D, Sprafka M.The smoking habits of Minnesota physicians.Am J Public Health 1993;83(3):415-417.

11. Mengual L, Perula de Torres LA, Redondo J, Roldán A, Prada A, Martínez de la Iglesia J et al. Evolution in consumption of and attitude towards to bacco towards physicians at the regional hospital "Reina Sofía", Córdoba. Gac Sanit 1996;10(52):18-24.

12. Antoniu S. High levels of smoking in Romanian doctors. The Lancet 2000;356:1420

13.W orld Health 0 rganization. To bacco or Health:A global status report, 1997. Ginebra:W HO 19

14. Secretaría de Salud. El consumo de tabaco en México y Encuesta Nacional de Adicciones 1998 (Tabaco). México, D.F.:SSA, 2000.
15. Li H, Fish D, Zhou X. Increase in cigarette smoking and decline of antismoking counselling among Chinese physicians: 1987-1996. Health Promotion International 1999;14:123-131.

16. Tessier JF, Rene L, N ajjari C. Attitudes and opinions of french general practitioners towards to bacco. Tob Control 1993;2:226-230.

17. Zanetti F, Gambi A, Bergamaschi A, G entilini F, De Luca G, Monti C et al. Smoking habits, exposure to passive smoking and attitudes to a nonsmoking policy among hospital staff. Public Health 1998;112:57-62.

18. Dekker H, Looman C, Adriaanse H,Van Der Maas P. Prevalence of smoking in physicians and medical students, and the generation effect in the N etherlands. Soc Sci Med 1993;36:817-822.

19.W aldrom I. Patterns and causes of gender differences in smoking. Soc Sci Med 1991:32:989-1005.

20. Molarius A, Parsens R, D obsen AJ, Evans A, Fortmann SP, Jamrozik K et al. Trend in cigarette smoking in 36 population from the early 1980 s to the mid-1990s: Findings from the W HO MO NICA Project. Am J Public Health 2001;91:206-212.

21. Fernández L, Sánchez M. Prevalencia de consumo de tabaco entre las médicas y las enfermeras de la comunidad de Madrid. Rev Esp Salud Publica 1999:73:355-364.

22. Zanetti F, G ambi A, Bergamaschi A, Gentilini F, De Luca G, Monti C et al. Smoking habits, exposure to passive smoking and attitudes to a nonsmoking policy hospital staff. Public Health 1998;112:57-62.

23. Roth LK, Taylor HS. Risks of smoking to reproductive health: Assessment of women's knowledge. Am J 0 bstet Gynecol 2001;184:934-939.

24. N agle A, Schofield M, Redman S.Australian nurses' smoking behaviour, knowledge and attitude towards providing smoking cessation care to their patients. Health Promotion International 1999;14:133-144.

25. Lancaster T, Stead L, Silagg $C$ et al for the Cochrane Tobacco Addiction Review Group. Effectiveness of interventions to help people stop smoking findings from the Cochrane Library. BMJ 2000;321:355-358.

26. Meenan RT, Stevens VJ, Hornbrook MC, La Chance PA, Glasgow RE, Hollis JF et al. Cost-effectiveness of a hospital-based smoking cessation intervention. Med Care 1998:36:670-678.

27. Dickinson JA. US has placed tobacco imports to $C$ hina high on priority list for liberalisation. BMJ 2000;321(7273):1413-1414.

28. Cornejo E, Medina E, Mascaro J, Matus P, Muñoz JC, Castillo P. Actitudes y conductas respecto al uso de tabaco entre médicos de Santiago. Rev Med Chil 1994;122(9):1087-1094.

29. Bener A, Gomes J, Anderson JA. Smoking habits among physicians in two Gulf countries. J R Soc Health 1993;113:298-301.

30. MirraAP, Rosemberg J. Survey on prevalence of smoking among Brazilian physicians. Rev Assoc Med Bras 1997;43:209-16.

31. Hussain SF,Tjeder-Burton S, C ampbell IA, D avies PD.Attitudes to smoking and smoking habits among hospital staff.Thorax 1993;48:174-175.

32. Hay D R. Cigarette smoking by N ew Zealand doctors and nurses: Result from the 1996 population census. N Z Med J 1998;111:102-105.

33. Labrandero M, Escandón C, Ramírez J, Hernández J, Coreño 0. Consumo de tabaco entre médicos y enfermeras. Rev Med Inst Mex Seguro Soc 1995;33:55-60.

34. Sansores R, Ramírez A,Villalba J, Herrera L, Soriano A. Tabaquismo en médicos mexicanos. Un análisis comparativo con fumadores que no son médicos. Rev Invest Clin 2000;54(2):161-167.

35. Tapia-C onyer $\mathrm{R}, \mathrm{C}$ ravioto $\mathrm{P}, \mathrm{D}$ e la Rosa B, G alván F, G arcía de la Torre $G$, Kuri P. Cigarrette smoking; knowledge and attitudes among Mexican physicians. Salud Publica Mex 1997;39(6):507-512.

36. Ramírez-Casanova M, González E, 0 campo A, Sánchez S, Cicero R. Hábito tabáquico en trabajadores de un $\mathrm{H}$ ospital $\mathrm{G}$ eneral. Informe de una encuesta. Gac Med Mex 1991;127(3):283-286.

37. Peña-C orona M, Díaz G, Mondragón M, Zárate A. C onsumo de tabaco en trabajadores del IMSS. En: SSA, ed. El Consumo de tabaco en México y Encuesta $\mathrm{N}$ acional de Adicciones 1998 (Tabaco). México, D.F.: SSA, 2000: 71-77. 
38. Gómez-García R, Grimaldi-Carpio A. Tabaquismo en el personal de salud: estudio de una unidad hospitalaria. Salud Publica Mex 1998;40(1): 53-57.

39. Rojo-Padilla J, Borunda 0, Herrera-Moro J. Encuestas en médicos y paramédicos del ISSSTE. En: SSA, ed. El C onsumo de tabaco en México y Encuesta $\mathrm{N}$ acional de Adicciones 1998 (Tabaco). México 2000:79-85.

40. Fernández IH, Escobedo de la Peña J, Hernández-Tamayo D, TudónGarcés H, Ramírez-Galindo D, Benítez-Martínez MG et al. Consumo de tabaco en la población derechohabiente del Instituto Mexicano del Seguro Social. Salud Publica Mex 1997;39:125-132.
41. Garfinkel L, Stellman SD. Cigarette smoking among physicians, dentist and nurses. Cancer J C lin 1986;36:2-8.

42. W ells J, English P, Posner S, W agebknecht L, Perez-Stable E. Misclassification rates for current smokers misclassified as nonsmokers. Am J Public Health 1998:88:1503-1593.

43.W orld Health 0 rganization.Tobacco C ontrol Research. G eneva:W HO, July 1999.

44. A rillo E, Lazcano E, Peris M, Salazar E, Salmerón J, Alonso P. El conocimiento de profesionales de la salud sobre la prevención del cáncer cervical.Alternativas de educación médica. Salud Publica Mex 2000;42:34-42. 